\title{
Decreased white matter fractional anisotropy is associated with poorer functional motor skills following spinal cord injury: a pilot study
}

\author{
Erin E. Cunningham ${ }^{1} \cdot$ Jeremy W. Noble ${ }^{1} \cdot$ Andrei Krassioukov $^{2,3} \cdot$ Lara A. Boyd $^{4,5} \cdot$ Janice J. Eng ${ }^{2,4,6}$
}

Received: 7 May 2018 / Revised: 31 July 2018 / Accepted: 14 August 2018 / Published online: 5 September 2018

(c) International Spinal Cord Society 2018

\begin{abstract}
Study design Prospective cross-sectional study

Objectives The objective of this study was to assess associations between white matter changes and functional motor markers including grip strength and prehension in the upper limb.

Setting Single Center Imaging Study, in Vancouver Canada.

Methods Diffusion tensor imaging produced FA (Fractional Anisotropy) maps of the brain for participants with SCI $(n=7)$ and controls $(n=6)$. These FA maps were analyzed using tract-based spatial statistics. Correlations between the FA values (of the genu of the corpus callosum, the left superior longitudinal fasciculus and the right anterior thalamic radiation) of the SCI group and functional outcomes (grip strength, Graded Redefined Assessment of Strength, Sensibility and Prehension (GRASSP)) were assessed.

Results Significant differences $(p<0.05)$ were found between the FA values of the controls and the SCI group in two white matter clusters, with lower values in the SCI group. Strong correlations were found between the FA values of the identified clusters and the age of SCI participant, and the right GRASSP Quantitative Prehension and right total GRASSP score.

Conclusions This preliminary data suggests that decreased FA in the genu of the corpus callosum may be a biomarker for functional motor ability of the upper limb with higher FA indicating better ability. Further research needs to be done to determine if other white matter tracts are also associated with strength and use of the hand following SCI.

Sponsorship The International Collaboration on Repair Discoveries (operating grant) and Canada Research Chair Program (for JJE) provided support for this research.
\end{abstract}

Janice J. Eng

Janice.Eng@ubc.ca

1 Faculty of Kinesiology, University of New Brunswick, Fredericton, NB, Canada

2 International Collaboration on Repair Discoveries, Vancouver, BC, Canada

3 Department of Medicine, University of British Columbia, Vancouver, BC, Canada

4 Department of Physical Therapy, University of British Columbia, Vancouver, BC, Canada

5 Djavad Mowafaghian Centre for Brain Health, University of British Columbia, Vancouver, BC, Canada

6 Neurorehabilitation Research Program, G.F. Strong Rehabilitation Centre, Vancouver, BC, Canada

\section{Introduction}

Spinal cord injuries (SCI) have long been known to have the potential to impair movement and have other serious effects following the initial injury. How the brain responds to this devastating injury can be studied with Magnetic Resonance Imaging (MRI) as physiological changes within the brain can be linked with functional impairment and movement changes following SCI.

Diffusion tensor imaging (DTI), a MRI tool, can also be useful in the study of cerebral changes following SCI. Currently most research has focused on fractional anisotropy (FA) within the white matter tracts of the spinal cord following SCI. Petersen and colleagues found that FA was lower in multiple regions of interest (ROIs) within the spinal cord, in thoracic and cervical regions, when comparing individuals with SCI to controls [1]. Corticospinal tracts (CST) and sensory tracts have also showed lower FA 
values following SCI [1]. Additionally, findings have shown that individuals with a cervical SCI have lower FA in the cervical spinal cord than controls [2].

Ilvesmäka and colleagues used DTI metrics to compare the cerebral white matter integrity of people with SCI to healthy controls; they found that people with SCI had significantly lower FA and higher mean diffusivity (MD) than controls [3]. While previously, several studies had focused on the decreased FA following SCI in the CST [4, 5], Ilvesmäka et al. [3] found that association fibers (i.e. fasciculi), commissural fibers (i.e. the corpus callosum) and projection fibers (i.e. thalamocortical projections) all showed differences in DTI metrics, and therefore white matter integrity following SCI. This research is consistent with previous work that found following other neurological trauma such as stroke and schizophrenia [6, 7].

The brain reorganizes following SCI to compensate for loss of certain functions and injury to certain structures, as is indicated in a literature review by Kokotilo, Eng and Curt [8]. Although this has been suggested by many functional MRI (fMRI) studies, less is known about the mechanism behind these cerebral shifts [9]. Clinical scores (e.g. time since injury, severity of injury) correlate with decreased cerebral and spinal cord FA [1,3] and other research has linked abnormal FA in the spinal cord to altered motor connectivity and function [2]. It is however unclear if cerebral white matter changes, as is shown in DTI studies, are currently linked to functional ability such as movement or strength.

The current study used DTI derived tract based spatial statistics (TBSS) to examine the difference in cerebral white matter tracts between healthy controls and people with cervical SCI. An advantage of this approach is that it is hypothesis free and not constrained to ROI identified a priori. Additionally, the relationship between motor-sensory ability in the form of strength, prehension, and sensation in the upper limb, were examined relative to cerebral DTI metrics. It was hypothesized that FA would be significantly decreased in the brain areas identified by TBSS following SCI. Additionally, it was hypothesized that reductions in FA would correlate to motor-sensory ability.

\section{Methods}

\section{Participants}

A total of 14 community-living participants were recruited for the current study. The mean age of the participants was $44.9 \pm 9.2$ years $( \pm$ standard deviation $)$. Ten of the participants were male, while four were female. Two groups were formed from the 14 participants: a control group $(n=7)$ recruited from the community and a group of individuals
Table 1 SCI and control group demographics

\begin{tabular}{llllll}
\hline Participant & $\begin{array}{l}\text { Age } \\
\text { (years) }\end{array}$ & Sex & $\begin{array}{l}\text { Time } \\
\text { since } \\
\text { injury } \\
\text { (months) }\end{array}$ & $\begin{array}{l}\text { Neurological } \\
\text { level of injury }\end{array}$ & $\begin{array}{l}\text { American } \\
\text { spinal injury } \\
\text { scale score } \\
\text { (AIS) }\end{array}$ \\
\hline SCI-01 & 42 & M & 326 & C4-5 $^{\text {a }}$ & B \\
SCI-02 & 58 & M & 112 & C6 & A \\
SCI-03 & 54 & F & 234 & C4-5 & B \\
SCI-04 & 32 & M & 126 & C5-6 & C \\
SCI-05 & 41 & F & 208 & C7 & A \\
SCI-P07 & 45 & M & 320 & C5-6 & B \\
SCI-07 & 37 & M & 251 & C6-7 & B \\
\hline
\end{tabular}

${ }^{a}$ Neurological level was obtained through medical records, even when two levels were specified

who had experienced a SCI $(n=7)$ and had received treatment within the Vancouver Coastal Health region. There was an interruption in the DTI scanning for one of the participants in the control group, resulting in their data being removed from the study, therefore there were a total of six participants in the control group, and seven in the SCI group. The control group was age ( \pm 4 years) and sex matched to the SCI group and did not have any major medical or health conditions.

To be eligible for the current study, individuals with SCI were diagnosed with complete or incomplete SCI between $\mathrm{C} 4$ and $\mathrm{C} 7$ and experienced tetraplegia (Table 1). All injuries were considered traumatic. The neurological level and completeness of the injury were self-reported by the participant and verified through medical records, including the American Spinal Injury Association (AIS) classification. While some participants were aware of the neurological level and completeness of their injury, most were not and this information was obtained from medical records from the local health authorities. The level stated in the medical record was used, even if two adjacent levels were stated. We did not do an examination to determine the neurological level of injury and the completeness of injury.

Participants in the SCI group were required to have some control over wrist movement, while having some hand and finger impairment. All participants were right hand dominant prior to the injury. The mean number of months since injury was $225.4 \pm 84.3$ (range 112-326). The mean age of the group with SCI was 44.1 years $( \pm 9.2)$ with a range of 32-58 years old. Two participants in this group were females, while five were males.

The control group had a mean age of 45.6 years $( \pm 9.8)$ with a range of 35-55 years old. Two controls were female, while the other four were males.

Participants in both groups were screened to ensure there were no contraindications for MRI scanning. This required extensive chart reviews for the SCI to ensure that any 
devices implanted at the time of injury were safe for MRI scanning. The sample size was limited to this exploratory group by resources to secure scanning time and the ability to find individuals in the SCI group that met the inclusion criteria.

All participants gave informed consent and both the University of British Columbia and local health authorities (Vancouver Coastal Health and Fraser Health) ethics review boards approved the procedures.

\section{Experimental design}

All participants attended an orientation session prior to MRI scanning. During this session written consent was obtained. Individuals with SCI were also evaluated with the Graded Redefined Assessment of Strength, Sensibility and Prehension (GRASSP). The GRASSP was administered by a certified Physical Therapist, who had experience working with an SCI population. The GRASSP identifies both sensory and motor capability of the upper limb following cervical SCI and results in a total score, in addition to subscores of strength, prehension and sensation in both upper limbs $[10,11]$. It has been shown that the GRASSP test has strong test-retest reliability and validity [12].

Following the orientation session, all participants took part in an MRI session.

\section{MRI data collection}

All MRI images were acquired at the University of British Columbia $3 \mathrm{~T}$ MRI Research Centre. A Philips Gyroscan Intero $3.0 \mathrm{~T}$ whole-body scanner, equipped with a 16-channel head coil, was used to collect all MRI images (Philips, Best, the Netherlands). A T1-weighted anatomical scan of the brain was done for each participant (170 axial slices, matrix size $=$ $256 \times 256$, voxel size $=1.0 \times 1.0 \times 1.0 \mathrm{~mm}, \quad \mathrm{TE}=5 \mathrm{~ms}$, $\mathrm{TR}=24 \mathrm{~ms}$, Flip Angle $=40^{\circ}$ ).

DTI images were obtained with a single shot spin-echo DW imaging sequence $(\mathrm{TR}=7465 \mathrm{~ms}, \mathrm{TE}=60 \mathrm{~ms}, \mathrm{FOV}=$ $212 \times 1312 \mathrm{~mm}, 60$ slices, $2.2 \mathrm{~mm}$ slice thickness, voxel dimensions $=2.2 \mathrm{~mm}^{3}$ ). Diffusion weighting was applied across 15 non-collinear orientations $\left(b=1000 \mathrm{~s} / \mathrm{mm}^{3}\right)$, along with a non-weighted diffusion image $(b=0)$.

Additional echo-planar imaging (EPI) images were obtained during rest and during a functional movement task involving the wrist for separate analysis not described in this paper.

\section{Tract based spatial statistics analysis}

FMRIB Software Library (FSL v5.0) was used for preprocessing, processing, and analysis steps [13]. Participants without the complete datasets necessary for the DTI analysis were excluded from the study; this caused the exclusion of one control participant.

\section{Preprocessing}

All MRI datasets were converted to necessary formats using MRICron (http://people.cas.sc.edu/rorden/mricron/index. html). Following conversion, eddy current corrections were run in FSL to correct for head movement and distortions caused by in vivo functions such as cardiac and respiratory activity, on the datasets of all participants. The brain extraction tool, using the corrected dataset for each participant, then created a brain mask [14]. FSL's DTIfit was used to create single FA datasets for each participant by fitting tensors to the files. All results were then visually inspected to ensure no abnormalities.

\section{Processing}

TBSS methods, in FSL, were then applied to all participants' data, following all standard processing steps, to allow for DTI analysis [15, 16]. TBSS involves a whole-brain voxelwise analysis of FA values [15]. Nonlinear transformations were applied to the FA images to create a standard space across all participants. The standardized FA maps of the participants were combined into a 4D image, and a mean FA map of the sample was created for statistical analysis. The threshold was applied to the mean FA map with an FA value of 0.2 to find common areas of high FA values across participants. Each participant's FA map was then projected against the mean FA map to generate voxelwise comparisons of the participant to the mean. In order to find differences between the two groups, two-sample $t$-tests were run comparing the FA datasets of the control participants to those of the SCI group; this was done using the randomize tool in FSL, with 5000 random permutations. A significance level of $p<0.05$ was used, with a correction for multiple-comparisons using threshold-free cluster enhancement [17]. An image was produced to show areas of statistically significant differences between the two groups. This $t$-statistic image was edited to fill the regions showing significant differences, in order to produce a more useful image for further analysis. Clusters of voxels greater than 100 voxels in size that showed significant differences between the control and SCI groups were identified and were used as ROIs for comparisons of FA values. The results of these analyses are shown in Fig. 1.

Results of the FA value analysis and GRASSP were checked for normality with the Shapiro-Wilk test, and were all found to be normally distributed. The mean FA values from clusters identified in the TBSS analysis were compared between the control group and the SCI group using two-sample $t$-tests, which were undertaken using $\mathrm{R}$ (version 


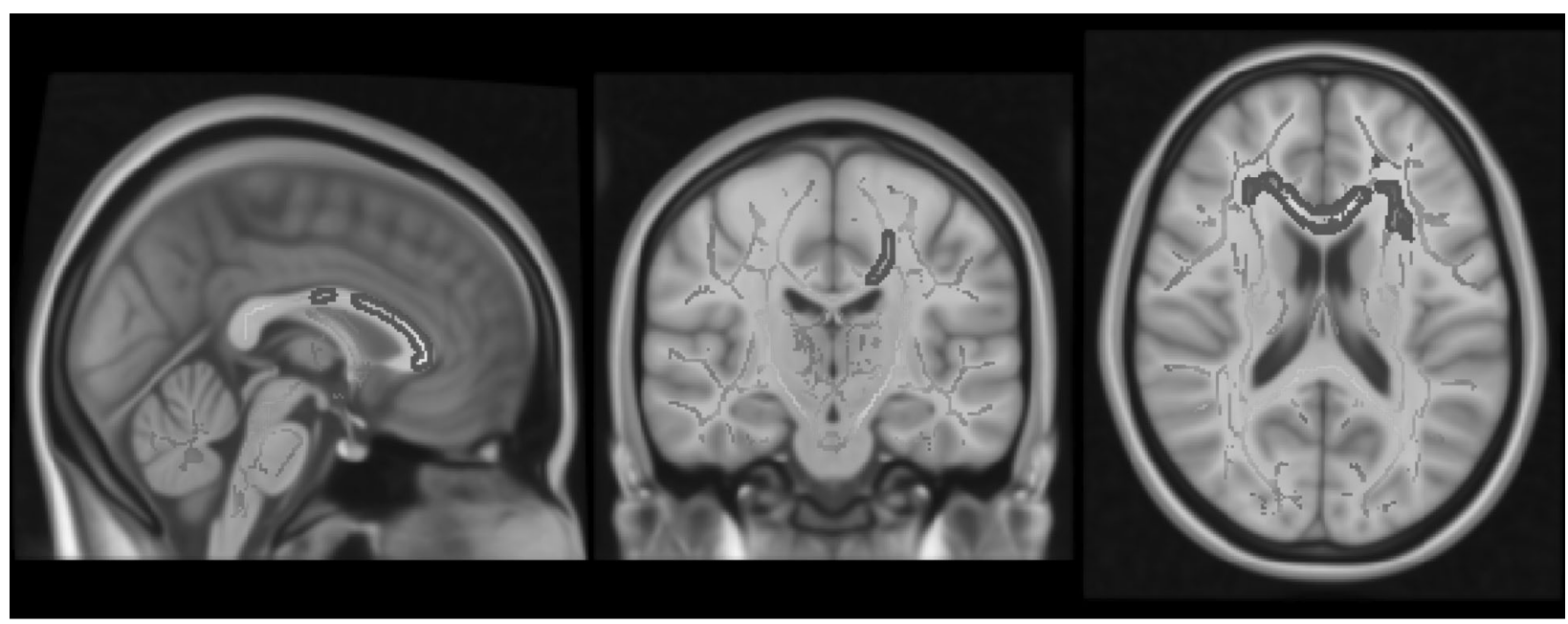

Fig. 1 Overlaid skeletonized and $t$-statistic images on an anatomical brain (MN152_T1_1mm). The same layout was used for each participant

Table 2 Grip strength and GRASSP values for the group with SCI, as well as grip strength values for the control group. All values are reported $\mathrm{R} / \mathrm{L}$

\begin{tabular}{llllllll}
\hline Participant & $\begin{array}{l}\text { Grip } \\
\text { strength } \\
(\mathrm{kg})\end{array}$ & $\begin{array}{l}\text { GRASSP } \\
\text { strength/50 }\end{array}$ & $\begin{array}{l}\text { GRASSP } \\
\text { SWM } \\
\text { dorsal/12 }\end{array}$ & $\begin{array}{l}\text { GRASSP } \\
\text { SWM } \\
\text { palmar/12 }\end{array}$ & $\begin{array}{l}\text { GRASSP } \\
\text { prehension- } \\
\text { qualitative/ } \\
12\end{array}$ & $\begin{array}{l}\text { GRASSP } \\
\text { prehension- } \\
\text { quantitative/ } \\
30\end{array}$ & $\begin{array}{l}\text { GRASSP } \\
\text { total score/ } \\
116\end{array}$ \\
\hline SCI-01 & $0 / 0$ & $20 / 17$ & $8 / 2$ & $6 / 5$ & $3 / 2$ & $14 / 12$ & $51 / 38$ \\
SCI-02 & $0 / 0$ & $19 / 19$ & $5 / 5$ & $8 / 7$ & $0 / 0$ & $6 / 6$ & $38 / 37$ \\
SCI-03 & $0 / 0$ & $11 / 13$ & $12 / 10$ & $12 / 12$ & $2 / 2$ & $10 / 6$ & $47 / 43$ \\
SCI-04 & $8.67 / 8.67$ & $16 / 24$ & $9 / 9$ & $10 / 12$ & $1 / 8$ & $11 / 18$ & $47 / 71$ \\
SCI-05 & $0 / 0$ & $26 / 17$ & $5 / 7$ & $6 / 6$ & $0 / 0$ & $14 / 9$ & $51 / 39$ \\
SCI-06 & $0 / 0$ & $15 / 16$ & $2 / 5$ & $4 / 4$ & $3 / 2$ & $14 / 13$ & $38 / 40$ \\
SCI-07 & $4 / 35.6$ & $34 / 45$ & $10 / 10$ & $12 / 11$ & $9 / 12$ & $25 / 30$ & $90 / 108$ \\
CON-01 & $28.3 / 27$ & & & & & & \\
CON-02 & $52 / 55.3$ & & & & & & \\
CON-03 & $51 / 45$ & & & & & & \\
CON-04 & $37 / 38.6$ & & & & & & \\
CON-05 & $48 / 41$ & & & & & & \\
CON-06 & $40 / 41$ & & & & & & \\
\hline
\end{tabular}

3.3.3) [18] Correlational analyses were undertaken between the FA values of the SCI group and age, grip strength, overall GRASSP score, GRASSP strength, sensation and prehension sub-scores, and the time since injury. Pearson moment correlations were used when the clinical tests data were normally distributed. If the data were not normally distributed Spearman correlations were used.

\section{Results}

\section{Grip strength and GRASSP results}

The GRASSP results for the SCI group participants and the grip strength results for both the control and SCI group participants are reported in Table 2.

\section{Tract-based spatial statistics results}

Figure 1 illustrates the skeletonized white matter and voxels identified by the TBSS process. Two clusters were identified within the TBSS produced image and are shown in Fig. 2. These clusters were employed in the correlational analysis as each showed significantly lower FA readings in the SCI group than the control group $(p<0.05)$. Details regarding these clusters are in Table 3 . Cluster one, the largest of the three clusters identified, included the genu of the corpus callosum, and extended into other white matter regions in the anterior brain, specifically the anterior corona radiata. Both clusters two and three are portions of the body of the corpus callosum.

Equal variance $t$-tests between the two groups showed significant differences in the FA values of the clusters. A 


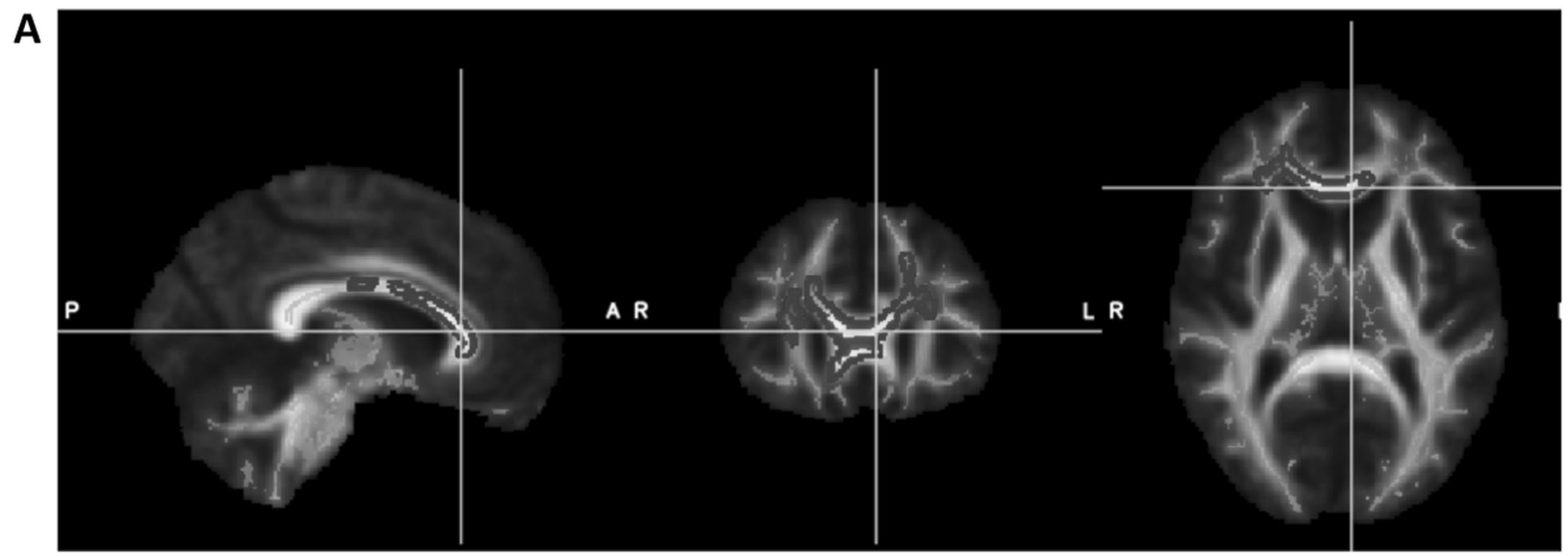

B

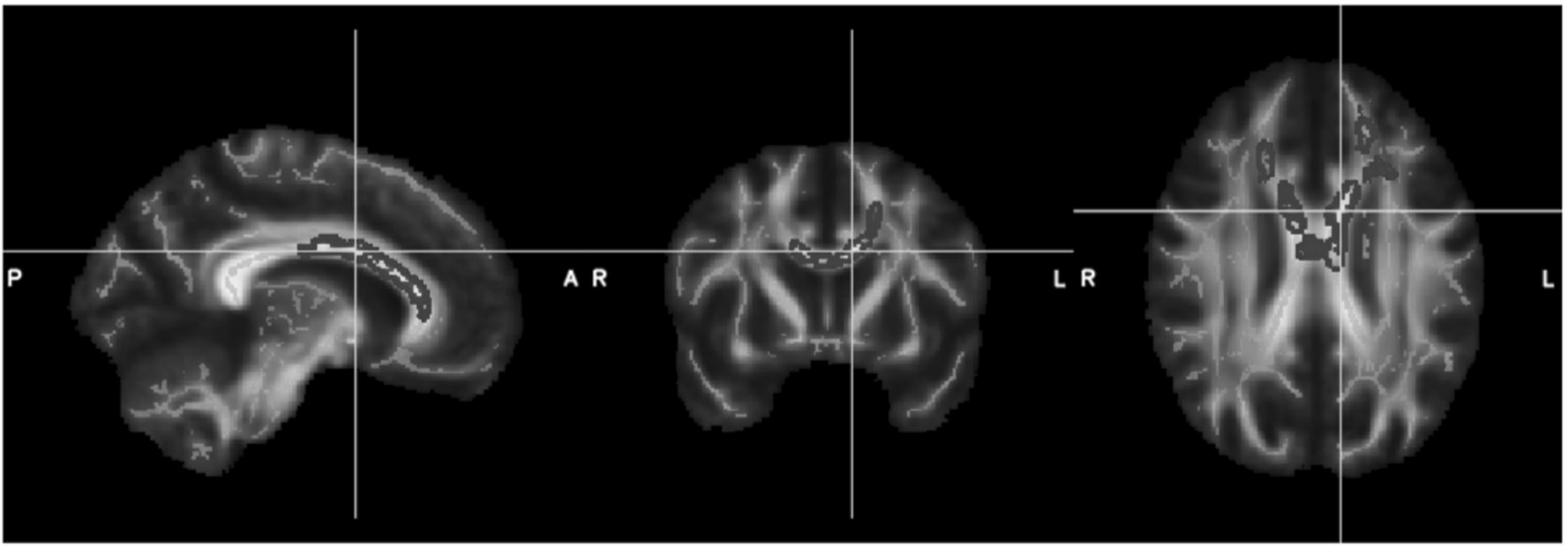

Fig. 2 Location of the two clusters identified by the TBSS process: a Genu of Corpus Callosum, b Body of the Corpus Callosum. The cross-hairs indicate the peak FA value within each cluster

Table 3 Location of clusters showing significant differences in FA between the SCI group and control group, identified using Tract-Based Spatial Statistics. Coordinates are in Montreal Neurological Institute (MNI) space

significant difference $(t(11)=3.25, p=0.008)$ was found between the two groups at cluster one. The SCI group had the significantly lower FA values (SCI: $0.59 \pm 0.037$, control group: $0.65 \pm 0.025)$. Similarly, cluster two showed significantly lower FA values in the SCI group than the control group (cluster one: $t(11)=2.55, p=0.027$, SCI: $0.33 \pm 0.051$, control group: $0.39 \pm 0.037$ )

\section{Correlation results}

Significant correlations were found between the age of the SCI group participants and the FA values of cluster one $(r(5)=-0.78, \quad p=0.039,95 \% \quad \mathrm{CI}: \quad[-0.96,-0.06])$. Additionally, the FA of cluster one was found to correlate with the GRASSP Quantitative Prehension score of the right hand $(r(5)=0.90, p=0.0054,95 \% \mathrm{CI}$ : [0.46, 0.99]).
Cluster two FA values had a significant correlation with the total GRASSP score of the right hand $(\rho(5)=0.92$, $p=0.0036,95 \%$ CI: [0.42,1.00]). Scatter plots of significant correlation tests are shown in Fig. 3.

\section{Discussion}

Our hypothesis that the SCI group would have reduced FA, as compared to the controls, was supported by the data. Additionally, decreased FA in the genu and body of the corpus callosum, as well as the anterior corona radiata, correlated with motor function, supporting the second hypothesis in this study. Quantitative prehension and overall GRASSP scores of the right hand were associated with decreased FA values in the first cluster. Included in this 
Fig. 3 Scatter plots of Significant Correlations between FA values and Demographic and Motor Skill Variables. Cluster 1 is the centered on Genu of the Corpus Callosum, while Cluster 2 is centered on the Body of the Corpus Callosum
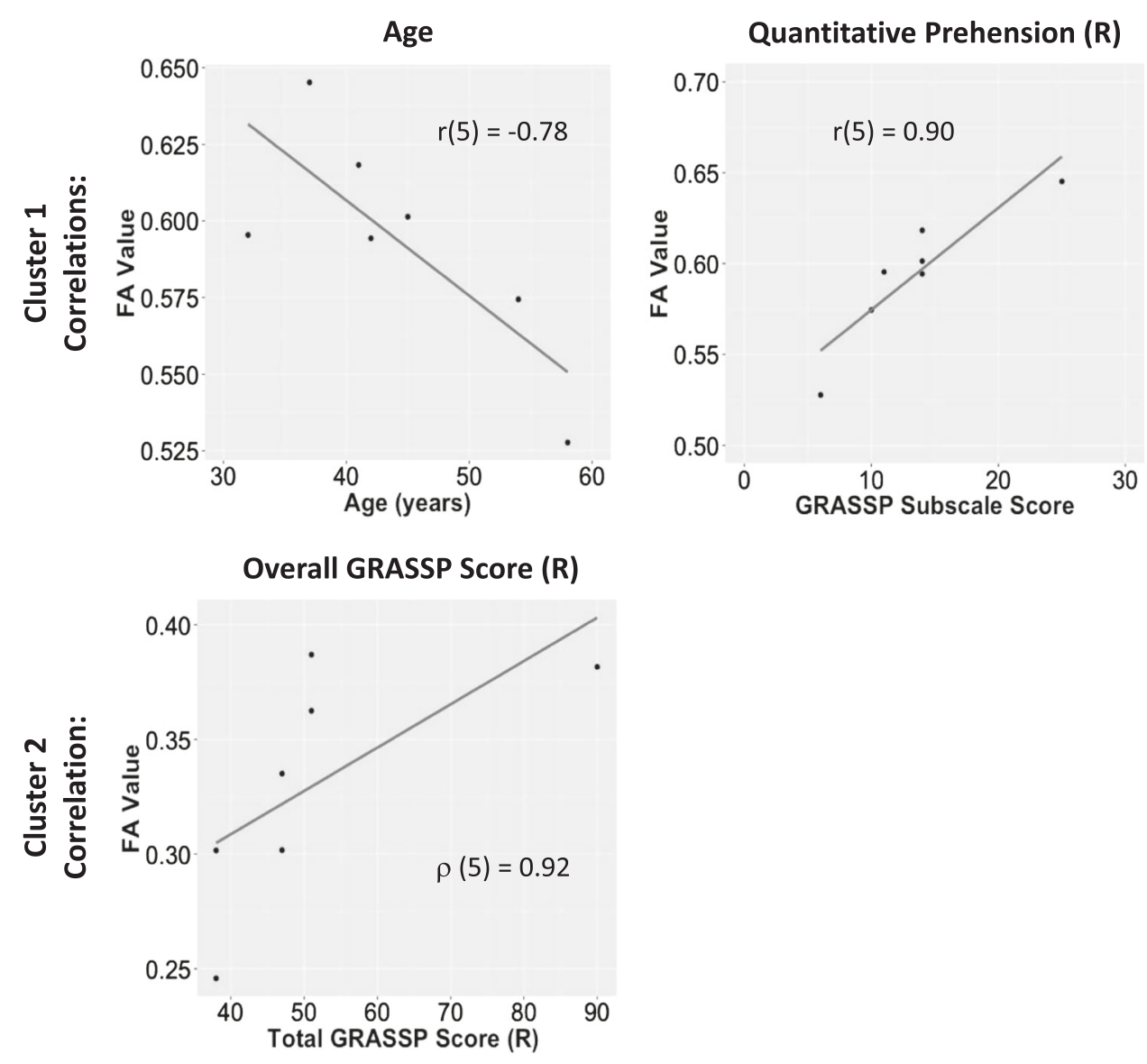

cluster, the corpus callosum functions to integrate sensory and motor signals between the left and right hemispheres of the brain [19]. The finding of decreased FA indicates that the decreased white matter integrity or demyelination, as shown by the reduced FA, may play a role in functional motor ability following SCI. As may be expected, higher FA values were found in participants with stronger functional ability, indicating a relationship between white matter tracts and motor function. Additionally, the anterior corona radiata is associated with the CST and the internal capsule [20]. This is the first study, to our knowledge, that links functional ability, as measured by the GRASSP test, to anatomical white matter changes in tracts such as the genu of the corpus callosum.

The findings of decreased FA following SCI support previous research [3]. Neurological trauma, such as stroke and SCI, has been shown to reduce FA in the corpus callosum, a finding which is consistent with the current study $[3,6]$. It has been suggested that the decreased FA may be caused by retrograde Wallerian degeneration, allowing for less restricted water diffusion in the cerebral white matter tracts [5]. It should also be mentioned that this change in FA may be due to compensatory mechanisms relative to motor impairment. It could be thought that the brain reorganizes following trauma such as SCI, to allow for maximal motor function. In doing so, areas of the brain that would not normally contribute to hand function (i.e. the genu of the corpus callosum) may become more involved as the compensatory pathways emerge [8]. In particular, this may occur due to the link between the genu of the corpus callosum and premotor and supplementary motor areas [21].

The correlation of motor ability with FA is consistent with previous studies as well, although this is the first to use a measurement of upper limb impairment and function. Koskinen et al. found that higher FA for the centrum semiovale was related to less impairment assessed by the International Standards for Neurological Classification of Spinal Cord Injury (ISNSCI) motor and sensory scores [22]. This is consistent with what was found in the current study, with higher FA values relating to better functional motor ability in the upper limb.

Previous research has shown that decreased FA in the centrum semiovale is related to motor and sensory scores of the INCSCI scale [22]; this is consistent with the current finding that decreased FA, and therefore decreased white matter integrity, in the anterior corona radiata is related to less motor function in the upper limb. It is not surprising that decreased motor function correlates with decreased white matter integrity in the CST and its associated areas, as 
this area plays a role in both sensory and motor function of the limbs.

The current study is also consistent with findings involving motor recovery following stroke. It has been shown that stroke patients with higher FA in the CST show better motor recovery [23, 24]. While SCI patients in the current study were examined a minimum of 112 months following the injury (i.e. in the chronic phase) and motor recovery is unlikely past the existent state, the suggestion that higher FA indicates better motor ability is similar to the current findings. It may be interesting for future research to examine individuals shortly after SCI (i.e. the acute phase) to determine if FA values are correlated to motor recovery.

Previous research has shown that handedness affects differences in FA values and white matter structure in the left and right hemispheres of the brain [25, 26]. This lateralization, also seen for language, would mean higher FA values are seen in the hemisphere opposite the dominant hand (i.e. the left for right-handed individuals) $[25,26]$. The current study consisted of participants who were all right-hand dominant. Thus prior to the SCI, it is possible that the participants with SCI had greater white matter organization on the left side of the brain; this would also be true for the controls. Following SCI, hand dominance decreases as functional ability changes. With this, it is thought that FA may decrease in the hemisphere that was associated with the dominant hand to resemble that of the opposite hemisphere (which would have been associated with the non-dominant hand). Since all of the participants in the current study were right-hand dominant, this may explain why relationships were found between the right hand and FA values, while no significant relationships were found in association with the left hand.

Previous work has shown that there is a decrease in FA that occurs with aging [27]. This decrease has been attributed to age decreases in myelination, and axonal shrinkage. The present study observed a negative correlation between age and FA within cluster 1 for the SCI group, but not the control group. It is possible that this relationship may be stronger in the SCI group due to the compounding effects of the SCI and gaining. It is likely a stronger correlation would have been observed in the control group if a wider range of ages were recruited.

While this research is merely preliminary findings, it may suggest that decreased FA in the genu of the corpus callosum and the anterior corona radiata is a biomarker for lower functional motor ability in the upper limb following SCI. This may prove useful in the design of future rehabilitation interventions that try to target these regions. Further research will need to investigate how this can be applied to recovery aspects of SCI.

\section{Limitations}

The current study was limited by a small sample size, requiring the use of a large alpha value $(p=0.05)$. The current study was limited by a small sample size. In addition, we did not correct the alpha for multiple comparisons due to the exploratory nature of the study, which may have resulted in a greater chance of type I error.

Additionally, it cannot be confirmed that participants with SCI had not suffered from a traumatic brain injury (TBI), although none were diagnosed. TBI has been shown to cause significant decreases in FA in the corpus callosum [26]. It is possible that participants had suffered a TBI at the time of the SCI, which may have caused decreased FA in the corpus callosum however none of the medical charts for the SCI group indicated any brain injury.

It should also be noted that DTI measures such as FA can be affected by crossing tracts in the white matter [28]. Although FA is not directionally dependent on the eigenvectors of DTI, this possibility should still be considered [28]. The significant spearman correlation for cluster 2 FA and total GRASSP score appears to be the result of two extreme cases, and therefore should be interpreted with caution.

\section{Conclusion}

Overall, functional motor ability of the right hand, as measured by the GRASSP test, was related to decreased FA in the genu of the corpus callosum following SCI. Further research with a larger sample size should be performed to determine if other white matter tracts also correlate with motor ability. Additionally, research should examine the acute phase SCI relative to FA and motor recovery potential. An investigation into motor recovery and hand dominance may also prove interesting.

Acknowledgements Assistance in collection of clinical tests and MRI data from Ms. Caryne Torkia and Ms. Chihya Hung is acknowledged. Discussion on DTI data processing techniques with Ms. Jennifer Ferris are also acknowledged.

Funding The International Collaboration on Repair Discoveries (operating grant) and Canada Research Chair Program (for JJE) provided support for this research.

Author contributions EEC was responsible for analyzing the DTI data from the study, and completing the statistical analysis, and contributed to the writing of the report. JWN was responsible for the study and protocol design. Additionally, he was responsible for the recruitment of participants, collection of data, and oversaw the DTI and statistical analysis and contributed to the writing of the report. AK assisted in the study design and was responsible for the clinical screening of the SCI group participants. He provided feedback on the report. LAB was responsible for study and protocol design and consulted on the analysis of the MRI data. She contributed to the writing of the manuscript. 
JJE was responsible for study and protocol design, oversaw the data collection process, and consulted on the data analysis protocols. She contributed to the writing of the manuscript.

\section{Compliance with ethical standards}

Conflict of interest The authors declare that they have no conflict of interest.

\section{References}

1. Petersen JA, Wilm BJ, von Meyenburg J, Schubert M, Seifert B, Najafi Y, et al. Chronic cervical spinal cord injury: DTI correlates with clinical and electrophysiological measures. J Neurotrauma. 2012;29:1556-66. https://doi.org/10.1089/neu.2011.2027

2. Chang Y, Jung T-D, Yoo DS, Hyun JK. Diffusion tensor imaging and fiber tractography of patients with cervical spinal cord injury. J Neurotrauma. 2010;27:2033-40. https://doi.org/10.1089/neu. 2009.1265

3. Ilvesmäki T, Koskinen E, Brander A, Luoto T, Öhman J, Eskola H. Spinal cord injury induces widespread chronic changes in cerebral white matter. Hum Brain Mapp. 2017;3647(April): 3637-47. https://doi.org/10.1002/hbm.23619

4. Freund P, Wheeler-Kingshott CA, Nagy Z, et al. Axonal integrity predicts cortical reorganisation following cervical injury. J Neurol Neurosurg Psychiatry. 2012;83:629-37. https://doi.org/10.1136/ jnnp-2011-301875

5. Guleria S, Gupta RK, Saksena S, et al. Retrograde Wallerian degeneration of cranial corticospinal tracts in cervical spinal cord injury patients using diffusion tensor imaging. J Neurosci Res. 2008;86:2271-80. https://doi.org/10.1002/jnr.21664

6. Borich MR, Mang C, Boyd LA. Both projection and commissural pathways are disrupted in individuals with chronic stroke: investigating microstructural white matter correlates of motor recovery. BMC Neurosci. 2012;13:107 https://doi.org/10.1186/1471-220213-107

7. Karlsgodt KH, Van Erp TG, Poldrack RA, Bearden CE, Nuechterlein KH, Cannon TD. Diffusion tensor imaging of the superior longitudinal fasciculus and working memory in recent-onset schizophrenia. Biol Psychiatry. 2008;63:512-518.

8. Kokotilo KJ, Eng JJ, Curt A. Reorganization and preservation of motor control of the brain in spinal cord injury: a systematic review. J Neurotrauma. 2009;26:2113-26. https://doi.org/10. 1089/neu.2008.0688.Reorganization

9. Freund P, Rothwell J, Craggs M, Thompson AJ, Bestmann S. Corticomotor representation to a human forearm muscle changes following cervical spinal cord injury. Eur J Neurosci. 2011;34:1839-46. https://doi.org/10.1111/j.1460-9568. 2011.07895.x

10. Velstra I-M, Bolliger M, Tanadini LG, et al. Prediction and stratification of upper limb function and self-care in acute cervical spinal cord injury with the graded redefined assessment of strength, sensibility, and prehension (GRASSP). Neurorehabil Neural Repair. 2014;28:632-42. https://doi.org/10.1177/ 1545968314521695

11. Kalsi-Ryan S, Curt A, Verrier MC, Fehlings MG. Development of the Graded Redefined Assessment of Strength, Sensibility and Prehension (GRASSP): reviewing measurement specific to the upper limb in tetraplegia. J Neurosurg Spine. 2012;17 (Suppl1):65-76. https://doi.org/10.3171/2012.6.AOSPINE1258
12. Kalsi-Ryan S, Beaton D, Curt A, et al. The graded redefined assessment of strength sensibility and prehension: reliability and validity. J Neurotrauma. 2012;29:905-14. https://doi.org/10.1089/ neu.2010.1504

13. Woolrich MW, Jbabdi S, Patenaude B, et al. Bayesian analysis of neuroimaging data in FSL 2 . Neuroimage. 2008;45:S173-S186. https://doi.org/10.1016/j.neuroimage.2008.10.055

14. Smith SM. Fast robust automated brain extraction. Hum Brain Mapp. 2002;17:143-55. https://doi.org/10.1002/hbm.10062

15. Smith SM, Jenkinson M, Woolrich MW, et al. Advances in functional and structural MR image analysis and implementation as FSL. Neuroimage. 2004;23:S208-S219. https://doi.org/10. 1016/j.neuroimage.2004.07.051

16. Smith SM, Jenkinson M, Johansen-Berg H, et al. Tract-based spatial statistics: Voxelwise analysis of multi-subject diffusion data. Neuroimage. 2006;31:1487-505. https://doi.org/10.1016/j. neuroimage.2006.02.024

17. Smith SM, Nichols TE. Threshold-free cluster enhancement: Addressing problems of smoothing, threshold dependence and localisation in cluster inference. Neuroimage. 2009;44:83-98. https://doi.org/10.1016/j.neuroimage.2008.03.061

18. R Core Team (2017). R: A language and environment for statistical computing. R Foundation for Statistical Computing, Vienna, Austria. URL https://www.R-project.org/.

19. Dissecting the white matter tracts: interactive diffusion tensor imaging teaching atlas. http://www.asnr2.org/neurographics/7/1/ 26/White\%20Matter\%20Tract\%20Anatomy/DTI\%20tutorial\% 201.html Accessed on 2017.

20. Ducreax, D. Connectopeida knowedge database: anterior corona radiata. (2014-25). http://www.fmritools.com/kdb/white-matter/ complex-fibers/anterior-corona-radiata/index.html Accessed on October 2017.

21. Hofer S, Frahm J. Topography of the human corpus callosum revisited - comprehensive fiber tractography using diffusion tensor magnetic resonance imaging. Neuroimage. 2009;32:989-94. https://doi.org/10.1016/j.neuroimage.2006.05.044

22. Koskinen EA, Hakulinen U, Brander AE, Luoto TM, Ylinen A, Öhman JE. Clinical corelates of cerebral diffusion tensor imaging findings in chronic traumatic spinal cord injury. Spinal Cord. 2014;52:202-8. https://doi.org/10.1038/sc.2013.163

23. Wen H, Alshikho MJ, Wang Y, et al. Correlation of fractional anisotropy with motor recovery in patients with stroke after postacute rehabilitation. Arch Phys Med Rehabil. 2016;97: 1487-95. https://doi.org/10.1016/j.apmr.2016.04.010

24. Hervé PY, Crivello F, Perchey G, Mazoyer B, Tzourio-Mazoyer $\mathrm{N}$. Handedness and cerebral anatomical asymmetries in young adult males. Neuroimage. 2006;29:1066-79. https://doi.org/10. 1016/j.neuroimage.2005.08.031

25. Buchel C, Raedler T, Sommer M, Sach M, Weiller C, Koch MA. White matter asymmetry in the human brain: a diffusion tensor MRI study. Cereb Cortex. 2004;14:945-51. https://doi.org/10. 1093/cercor/bhh055

26. Wei CW, Tharmakulasingam J, Crawley A, et al. Use of diffusion-tensor imaging in traumatic spinal cord injury to identify concomitant traumatic brain injury. Arch Phys Med Rehabil. 2008;89:S85-S91. https://doi.org/10.1016/j.apmr.2008.07.005.

27. Bennett IJ, Madden DJ, Vaidya CJ, Howard DV, Howard JH Jr. Age-related differences in multiple measures of white matter integrity: a diffusion tensor imaging study of healthy aging. Hum Brain Mapp. 2009;31:378-90. https://doi.org/10.1002/hbm.20872

28. Wheeler-Kingshott CAM, Cercignani M. About "axial" and "radial" diffusivities. Magn Reson Med. 2009;61:1255-60. https://doi.org/10.1002/mrm.21965 